\title{
Binding of Benzimidazole Compounds to Conidia of Pithomyces chartarum
}

\author{
By F. J. STUTZENBERGER AND J. N. PARLE \\ Ruakura Agricultural Research Centre, \\ Hamilton, New Zealand
}

(Received 20 March 1972)

\begin{abstract}
SUMMARY
Conidia of Pithomyces chartarum (Berk. \& Curt.) M. B. Ellis strain C rapidly bound benzimidazole (BIZ), thiabendazole (TBZ), furidazole (FUD), and 2phenyl benzimidazole (PBIZ) when mixed with aqueous solutions of the compounds at room temperature. Almost complete removal $(>95 \%)$ of the compounds from solution could be achieved by raising the spore/compound ratio in the reaction mixtures. The binding process was temperature-independent over the range of o to $65{ }^{\circ} \mathrm{C}$ and $\mathrm{pH}$-dependent (optimum at $\mathrm{pH} 4.5$ ). BIZ binding was more sensitive to variation of ionic strength than was binding of TBZ, FUD or PBIZ.

Binding of BIZ conformed to the Langmuir adsorption isotherm; binding of the substituted benzimidazoles conformed to the isotherm over only part of the concentration range tested. Analysis of the binding data indicated that BIZ was bound to a single class of sites on the spore surface while the substituted benzimidazoles were bound to two or more classes differing in their affinity. This adsorption may be the first in a series of events leading to inhibition of spore germination by benzimidazole derivatives.
\end{abstract}

\section{INTRODUCTION}

Benzimidazole (I,3-benzodiazole) has been shown to act as a growth inhibitor in a variety of living systems including yeasts and bacteria (Woolley, I944), viruses (Brown, 1952) and the developing frog embryo (Bieber \& Hitchings, 1955). Some of its derivatives, such as 2-(4-thiazolyl) benzimidazole (thiabendazole) and 2-(2-furyl) benzimidazole (furidazole), are highly toxic to a wide range of fungi (Edgington, Khew \& Barron, I97I). Thiabendazole is currently being used to control the growth of Pithomyces chartarum (Berk. \& Curt.) M. B. Ellis, the fungus causing facial eczema, a mycotoxicosis in New Zealand grazing stock (Sinclair \& Howe, I968; Campbell, I969).

During studies on the effect which benzimidazole and its derivatives have on Pithomyces chartarum conidia, we observed that conidia rapidly removed benzimidazole compounds from solution in what appeared to be an adsorptive process at the spore surface. Previous reports (Lowry \& Sussman, I956; Sussman, von Boventer-Heidenhain \& Lowry, 1957) have suggested that fungal spore surfaces serve as reservoirs of inhibitory cations (such as Polymyxin-B) which are adsorbed there and excluded from vital areas of the spores until germination is induced. Such a phenomenon might be the first in a series of events leading to inhibition of $P$. chartarum spore germination by benzimidazole derivatives. This paper describes the binding of four benzimidazole compounds to $P$. chartarum spore surfaces and some factors which influence the process. 


\section{METHODS}

Organisms. Pithomyces chartarum strain c was used throughout this work. This strain, a heavily sporing laboratory isolate obtained in 1958 , has been previously described in regard to sporulation (di Menna, Campbell \& Mortimer, 1970) and nature of the spore surface (Bertaud, Morice, Russell \& Taylor, I963).

Preparation of spores. Cultures for spore production were routinely grown in cottonplugged milk bottles $(570 \mathrm{ml})$ containing $75 \mathrm{~g}$ whole barley grains and $45 \mathrm{ml}$ distilled water. After autoclaving for $30 \mathrm{~min}$ at $\mathrm{I} 20^{\circ} \mathrm{C}$ the medium was inoculated with about I ml of spore suspension and incubated at $20^{\circ} \mathrm{C}$ for 4 weeks. Spores were removed from the medium by sieving them through a 12 -mesh wire screen. A dense spore suspension in water was prepared and sieved through a nylon mesh (approximately 42 -mesh) to remove the fine debris of the medium. Spores were then washed at least six times with distilled water in a Buchner funnel, dried overnight under vacuum to a moisture content of 37 to $55 \%$, and stored at room temperature in a silica gel desiccator. Spore viability (as measured by germ-tube production on potato dextrose agar) remained above $99 \%$ during the washing-drying process; after 2 weeks of storage, viability averaged $93.5 \%$.

Benzimidazole compounds. The four benzimidazole compounds used in these studies were: unsubstituted benzimidazole (BIZ), thiabendazole (TBZ), furidazole (FUD), and 2-phenyl benzimidazole (PBIZ). Stock solutions of BIZ were prepared in distilled water; solutions of the three substituted benzimidazoles were prepared in $0.01 \mathrm{M}-\mathrm{HCl}$ to aid solution.

Reaction of benzimidazole compounds with spores. Reaction mixtures containing various spore densities ( $\mathrm{r}$ to $10 \mathrm{mg} / \mathrm{ml}$ ) and concentrations of benzimidazole compounds ( 3 to $50 \mu \mathrm{g} / \mathrm{ml}$ ) were magnetically stirred at room temperature simultaneously with control spore suspensions containing no benzimidazoles. Spores were removed from samples by Millipore filters at various time intervals. There was no detectable adsorption of benzimidazole compounds on the Millipore filters as determined by measurement of u.v. extinction before and after filtration. Clarified reaction mixture filtrates were spectrophotometrically scanned in the u.v. range with control spore fluids as blanks. The decrease in concentrations of free benzimidazoles was determined by the decrease in the u.v. spectra, using a PerkinElmer model $\mathrm{I} 37$ spectrophotometer with a $\mathrm{I} \mathrm{cm}$ cell. Residual TBZ was also measured by the A.O.A.C. Method I (Horwitz, I970).

\section{RESULTS}

Measurement of the decrease in the absorption spectra of BIZ, TBZ, FUD, and PBIZ appeared to be a reliable method for determining the decrease in free concentrations of the compounds after mixing in a spore suspension. Fig. I illustrates the u.v. spectra of the four compounds in aqueous solution at $\mathrm{pH} 5.0$, compared with the spectra of filtered fluids after the spores had removed a portion of each compound from solution. The average standard deviation of this method was calculated to be $\pm 3.3 \%$. Residual TBZ measurement by this method correlated with the A.O.A.C. method within a range of $\pm 5.9 \%$. In routine assays, decreases in the concentrations of BIZ, PBIZ, TBZ and FUD were measured by decreases in peak extinction at $272,295,298$, and $306 \mathrm{~nm}$ respectively.

Rapid removal of benzimidazole compounds by spores. Both viable and heat-killed $\left(60{ }^{\circ} \mathrm{C}\right.$, $30 \mathrm{~min}$ ) spores rapidly removed any of the four benzimidazole compounds from solution. Representative examples of this rapid removal are given in Fig. 2. Most of the reaction 

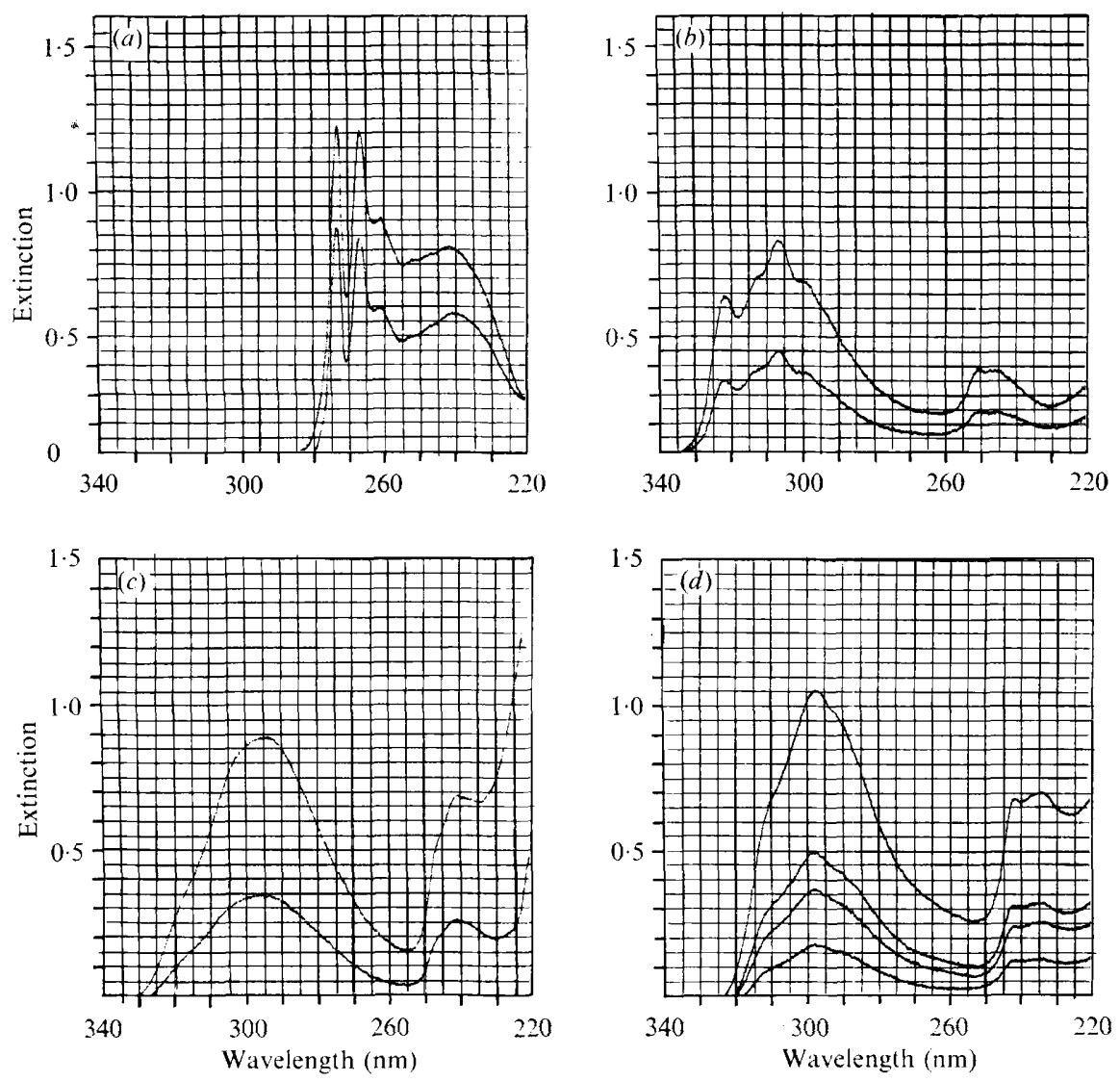

Fig. I. U.v. spectra of the four benzimidazole compounds in aqueous solution compared with the difference spectra of filtered reaction fluids: (a) BIZ $(20 \mu \mathrm{g} / \mathrm{ml}$, top tracing) compared with residual concentration after mixing with spores $(2 \mathrm{mg} / \mathrm{ml}) ;(b)$ FUD $(5 \mu \mathrm{g} / \mathrm{ml}$, top tracing) compared with residual concentration after mixing with spores $(0.25 \mathrm{mg} / \mathrm{ml}) ;(c)$ PBIZ (Io $\mu \mathrm{g} / \mathrm{ml}$, top tracing) compared with residual concentration after mixing with spores $(0.5 \mathrm{mg} / \mathrm{ml}) ;(d) \mathrm{TBZ}(\mathrm{IO} \mu \mathrm{g} / \mathrm{ml}$, top tracing) compared with residual concentrations after mixing with various spore densities $(0 \cdot 5, \mathrm{r} \cdot \mathrm{O}$, and $2.0 \mathrm{mg} / \mathrm{ml}$ in decreasing order). All reactions were run for $10 \mathrm{~min}$ in mixtures buffered to $\mathrm{pH} 5.0$ with $0.025 \mathrm{M}$-acetate.

( $>80 \%$ of maximum) occurred within the first min after mixing of the benzimidazoles and spores; the reaction appeared complete within 5 to $10 \mathrm{~min}$. The maximal amount of the benzimidazole compound removed from solution depended on the ratio of spore concentration (dry wt/ml) to benzimidazole compound concentration (Fig. 3). This ratio could be raised to the point at which $95 \%$ of the benzimidazole compound concentrations were removed from solution. Spore exudates did not appear to react with the compounds.

Unsubstituted benzimidazole is not toxic to spores even at $100 \mu \mathrm{g} / \mathrm{mg}$ dry wt of spores. Spores treated with FUD, TBZ or PBIZ remained viable through treatment and germinated when transferred to Sabouraud dextrose agar. However, germ-tube elongation was prevented at concentrations greater than $5 \mu \mathrm{g} / \mathrm{mg}$ dry wt spores.

Effect of temperature, $p H$, and ionic strength on adsorption of benzimidazole compounds. The characteristics of benzimidazole compound removal by spores suggested an adsorptive process at or near the spore surface. Variation of temperature over the range of o to $65{ }^{\circ} \mathrm{C}$ 


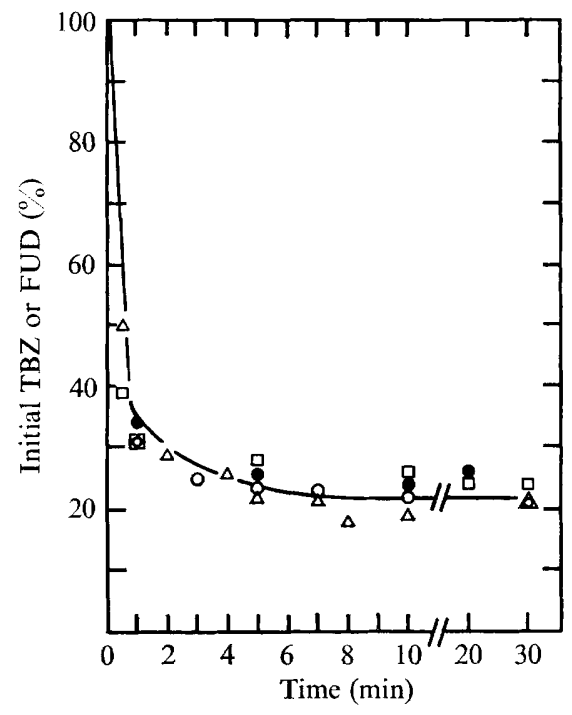

Fig. 2

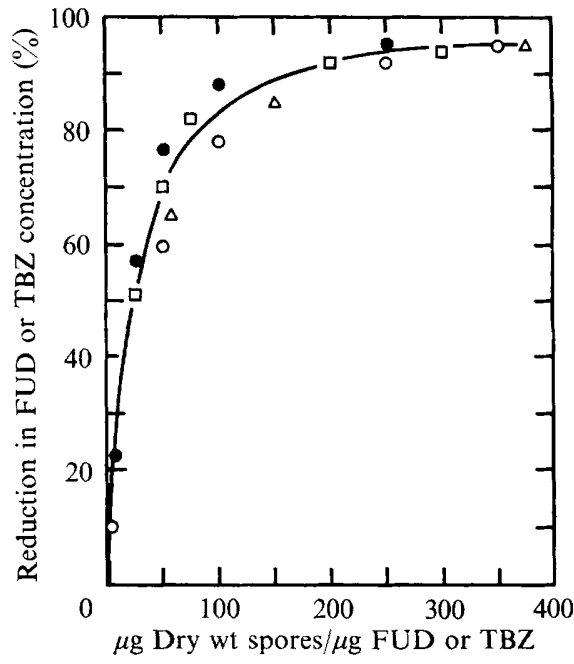

Fig. 3

Fig. 2. Time course of benzimidazole compound removal from solution after mixing with either viable or dead spores. $O$, TBZ with viable spores; $\triangle$, TBZ with dead spores; $\square$, FUD with viable spores;, FUD with dead spores. Reaction mixtures contained spores ( $\mathrm{mg} / \mathrm{ml}$ ), FUD or TBZ (10 $\mu \mathrm{g} / \mathrm{ml})$, and acetate buffer $\left(0.025 \mathrm{M}, \mathrm{pH} 5^{\circ} \mathrm{O}\right)$.

Fig. 3. Effect of varying the spore dry wt in reaction mixtures. $\bigcirc, \triangle, \square$, FUD concentrations of 2,4 , and $10 \mu \mathrm{g} / \mathrm{m} !$ respectively;, $\mathrm{TBZ}$ concentration of $5 \mu \mathrm{g} / \mathrm{ml}$.

had no detectable effect on the rate of extent of this adsorption, although temperatures above $35^{\circ} \mathrm{C}$ rapidly decreased spore viability. Adsorptive sites on the spore surfaces were remarkably heat-stable (spores heated at $97^{\circ} \mathrm{C}$ for 15 min retained $99 \%$ of their TBZbinding properties).

In determining the $\mathrm{pH}$ optimum for adsorption we found that the presence of some buffers had an interfering effect $(0.05 \mathrm{M}$-phosphate decreased benzimidazole adsorption about $30 \%$ ). Therefore reaction mixtures were made up to volume with varying amounts of $0.0 \mathrm{I} \mathrm{M}-\mathrm{HCl}$ or $0.0 \mathrm{I} \mathrm{M}-\mathrm{NaOH}$. After reaction, both the $\mathrm{pH}$ and the decrease in soluble benzimidazole compound were determined. The u.v. spectra of the benzimidazole compounds altered with variation in $\mathrm{pH}$. To overcome this change in spectra with $\mathrm{pH}$, all filtered samples were acidified to approximately $\mathrm{pH}_{\mathrm{I}} \cdot \mathrm{O}$ by addition to an equal volume of I $\mathrm{M}-\mathrm{HCl}$.

Fig. 4 illustrates the effect of $\mathrm{pH}$ on binding of benzimidazole compounds on spore surfaces. Maximal adsorption of all four compounds occurred at $\mathrm{pH} 4$ to 5 , with $50 \%$ of the maximal adsorption occurring at $\mathrm{pH}$ values of approximately $2 \cdot 6$ and $6 \cdot 8$. To determine whether decreases in adsorption at these latter $\mathrm{pH}$ values were due to irreversible inactivation of adsorptive sites on the spore surface, spore suspensions were adjusted to various $\mathrm{pH}$ values between 2.5 and 10.5 , stirred for $\mathrm{I} h$ at room temperature, then readjusted to $\mathrm{pH} 5.0$ and tested as to their adsorptive capacity. No irreversible decrease in adsorptive capacity occurred over the $\mathrm{pH}$ range tested.

The ionic strength of reaction mixtures was increased by the addition of $\mathrm{KCl}$ or $\mathrm{NaCl}$. Increases in ionic strength caused decreases in the adsorption of all four benzimidazole compounds; however, adsorption of the unsubstituted benzimidazole was the most affected 

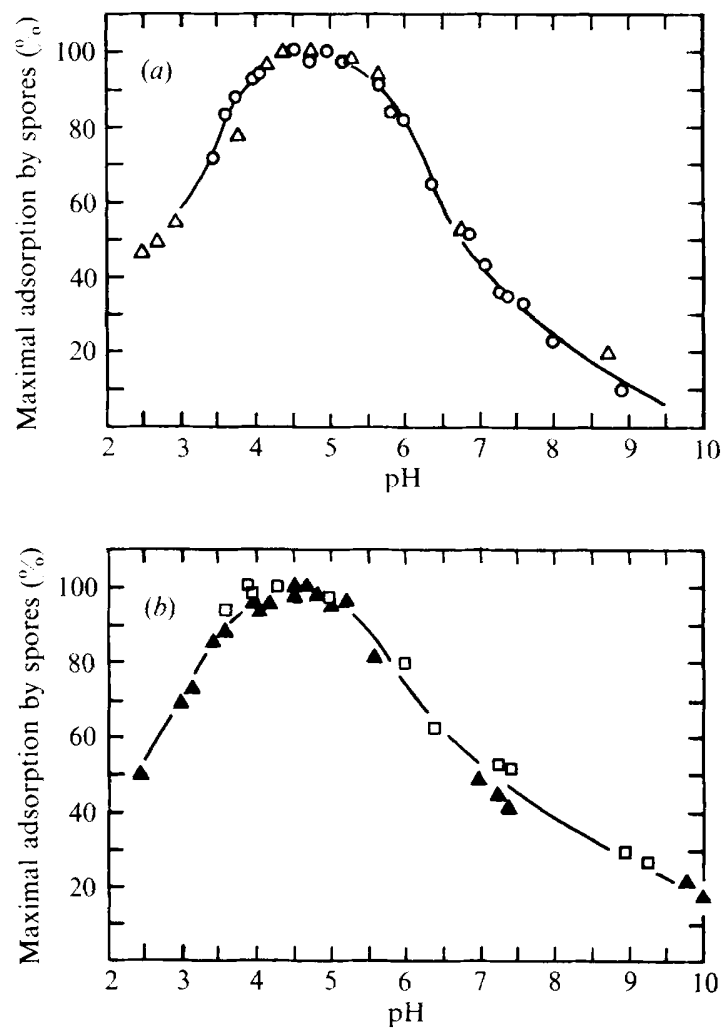

Fig. 4. Effect of pH on adsorption of benzimidazole compounds on spore surfaces. $\bigcirc, \mathrm{BIZ} ; \triangle$, PBIZ; $\square$, FUD; $\triangle$, TBZ. Points are the cumulative data of two or three experiments for each compound; the highest value obtained in each experiment was arbitrarily set at $100 \%$.

(Fig. 5). An increase to I M salt concentration caused a $70 \%$ decrease in the adsorption of the unsubstituted benzimidazole. Under similar conditions, adsorption of the substituted benzimidazoles decreased only II to $19 \%$. Likewise, the substituted benzimidazoles resisted elution with salt after they had been adsorbed by the spores, while elution of benzimidazole itself could be effected by raising the salt concentration.

Expression of the adsorption equilibrium as an isotherm. The equilibrium pertaining to the adsorption of a gas, liquid, or solute on to a solid surface is usually expressed as an adsorption isotherm. We wished to determine whether the adsorption of benzimidazole on spore surfaces conformed to a currently accepted theoretical isotherm. The amount of each benzimidazole compound adsorbed/mg dry wt of spores was determined over the maximal concentration range allowed by the limited solubility of the compounds. Conformity of data to the isotherm originally proposed by Langmuir (I9I6) was tested by the following equation:

$$
\frac{X_{f}}{X_{a}}=\frac{\mathrm{I}}{K X_{m}}+\frac{X_{f}}{X_{m}}
$$

where $X_{f}$ is the concentration $(\mu \mathrm{g} / \mathrm{ml})$ of free benzimidazole compound at equilibrium, $X_{a}$ is the amount adsorbed ( $\mu \mathrm{g} / \mathrm{mg}$ dry wt of spores), $X_{m}$ is the theoretical maximal amount adsorbed at infinite concentration and $K$ is the association constant for the system. This equation has been used by Hayward \& Trapnell (1964) to test conformity of data to the 

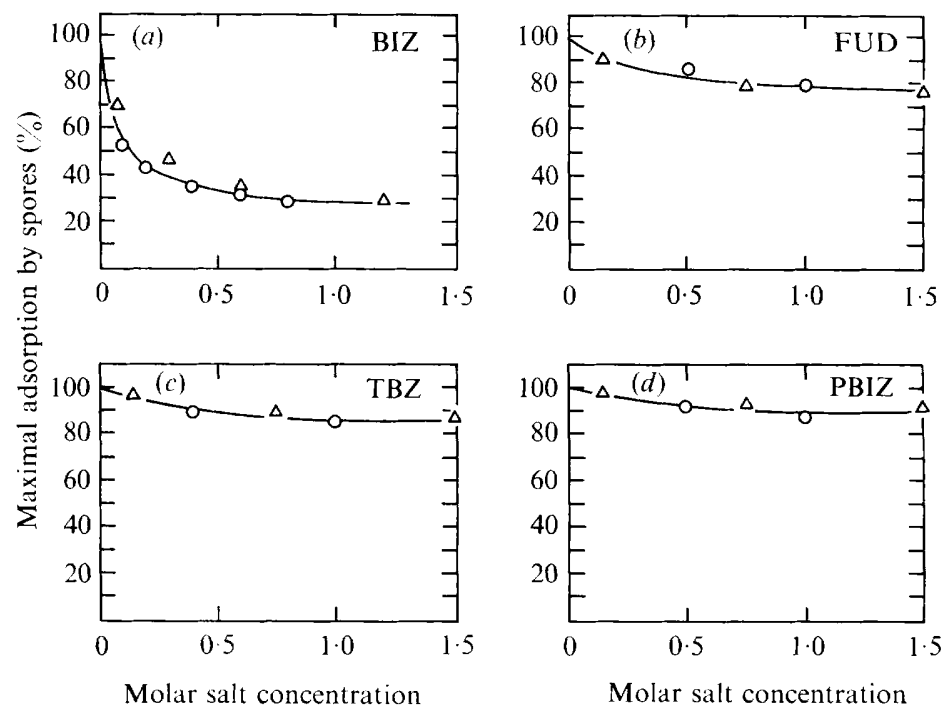

Fig. 5. Effect of ionic strength on adsorption of benzimidazole compounds on spore surfaces. $\mathrm{O}, \mathrm{KCl} ; \triangle, \mathrm{NaCl}$.
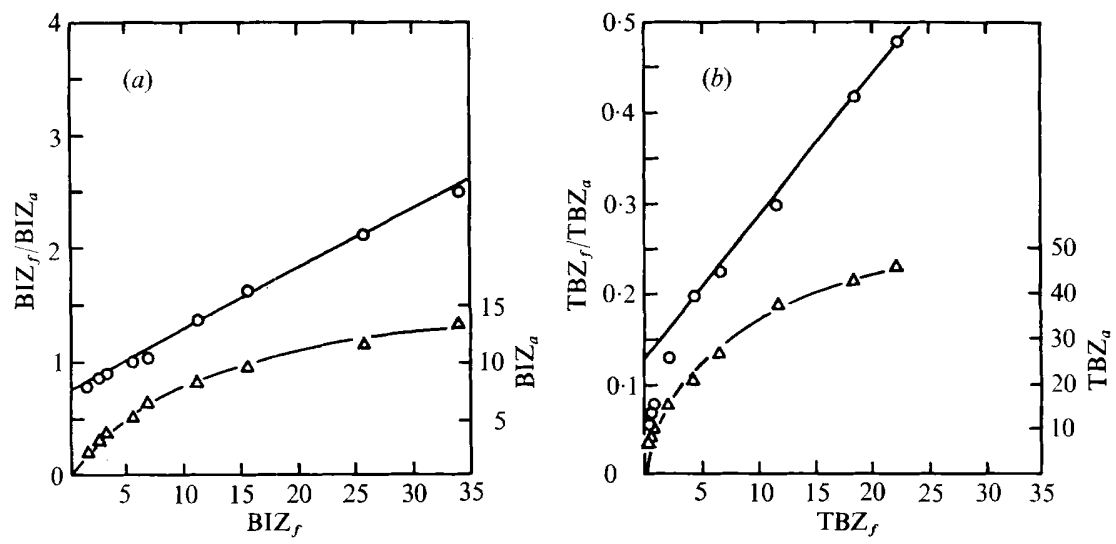

Fig. 6. Plots based on equation ( $\mathrm{I}$ ) testing conformity of BIZ and TBZ adsorption data to the Langmuir isotherm. $O, \mathrm{BIZ}_{f} / \mathrm{BIZ}_{a}$ and $\mathrm{TBZ}_{f} / \mathrm{TBZ}_{a} ; \triangle, \mathrm{BIZ}_{a}$ and $\mathrm{TBZ}_{a}$. Straight lines were fitted by the Linear Regression Method. Each point is the average of 2 to 4 determinations.

Langmuir isotherm: a plot of $X_{f} / X_{a}$ versus $X_{f}$ gives a straight line with a slope having the value of $\mathrm{I} / X_{m}$ if the system obeys the isotherm. Adsorption of BIZ conformed to the Langmuir isotherm over the complete range of values tested, while adsorption of TBZ, FUD and PBIZ conformed to the isotherm only at the higher $X_{f}$ values. Fig. 6 contains comparative plots for BIZ and TBZ to illustrate this difference. The $X_{m}$ values for BIZ and TBZ were 18.6 and 64.5 respectively. $X_{m}$ values obtained from similar plots for FUD and PBIZ adsorption were 63.2 and 105.2 respectively. These values, converted to $\mu \mathrm{mol}$ of benzimidazole compound adsorbed/mg dry wt of spores, were 0.15 for BIZ, 0.32 for TBZ, 0.34 for FUD and 0.54 for PBIZ.

The differences in $X_{m}$ values, together with the non-conformity to the Langmuir isotherm 

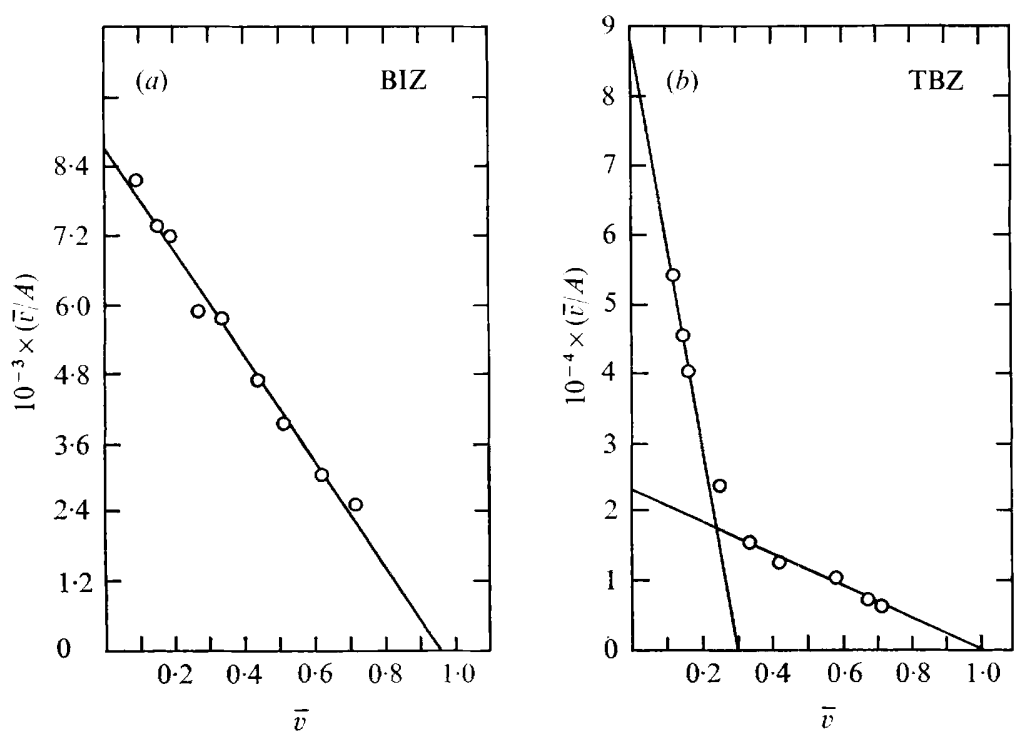

Fig. 7. Scatchard plots for BIZ and TBZ adsorption data.

Values for $A$ are expressed as mol/1 reaction mixture.

shown by the substituted benzimidazoles in the low concentration range, indicated differences in the number and type of sites binding TBZ, FUD and PBIZ as compared to those binding BIZ. Scatchard (1949) proposed an analysis, originally used in studies on the binding of small molecules by proteins, which was useful in detecting differences between benzimidazole compound binding classes. The basic equation in the analysis is:

$$
\vec{v}=\Sigma_{i} v_{i}=\Sigma_{i} \frac{n_{i} K_{i} A}{\mathrm{I}+K_{i} A},
$$

in which $\bar{v}$ is the average molar ratio of bound adsorbing molecules for the $i$ th binding site on the adsorbent, $n_{i}$ represents the fraction of the total number of binding sites which is contributed by the $i$ th class of sites, $K_{i}$ is the association constant for the interaction of this class of sites with the adsorbing molecules, and $A$ is the equilibrium concentration of unbound molecules in $\mathrm{mol} / \mathrm{l}$. The $\bar{v}$ values for the binding of each benzimidazole compound were calculated as $X_{a} / X_{m}$ using the $X_{m}$ values calculated from equation (I). These $\bar{v}$ values may also be regarded as the probability that any binding site, chosen at random from the reaction mixture, will have a molecule attached to it (Edsall \& Wyman, 1958).

For a single class of sites, equation (2) may be put into a form suitable for graphical analysis by solving for $K A$

and multiplying by $(\mathrm{I}-\bar{v}) / A$ to yield

$$
K A=\vec{v} /(\mathrm{I}-\vec{v}),
$$

$$
\bar{v} / A=K(\mathrm{I}-\bar{v}) \text {. }
$$

A plot of $\bar{v} / A$ versus $\bar{v}$ is linear if $K$ is constant. The intercept on the $\bar{v} / A$ axis is equal to $K n$, the slope equals $-K$, and the intercept on the $\bar{v}$ axis equals $n$. If binding involves two or more classes of sites, the plot becomes non-linear (Scatchard, I949).

When data for the binding of the benzimidazole compounds were analysed by the Scatchard method, two types of plots became apparent. Fig. 7 contains the plots for BIZ and TBZ as a comparison. The binding data for BIZ formed a linear plot; the association constant, $K$, was calculated to be $8.8 \times \mathrm{IO}^{3} \mathrm{l} / \mathrm{mol}$. The data for the three substituted benzimi- 
dazoles yielded inflected curves such as the one shown for TBZ binding. In this case, two classes of binding sites were assumed, one class having a high affinity for TBZ molecules, the other having a relatively low affinity. The $n$ values and $K$ values for each class of sites were calculated from the tangents of the TBZ curve by using the following set of equations:

$$
\begin{aligned}
n_{1} K_{1}^{2}+n_{2} K_{2}^{2} & =Y_{1}^{2} / \bar{v}_{1}, \\
n_{1}+n_{2} & =\bar{v}_{2}, \\
n_{1} K_{1}+n_{2} K_{2} & =Y_{1}, \\
n_{1} / K_{1}+n_{2} / K_{2} & =\bar{v}_{2}^{2} / Y_{1},
\end{aligned}
$$

where $Y_{1}$ and $Y_{2}$ are the upper and lower intercepts on the $\bar{v} / A$ axis respectively and $\bar{v}_{1}$ and $\bar{v}_{2}$ are the lesser and greater intercept values on the $\bar{v}$ axis respectively. These intercepts were determined by the extrapolation of the linear regression lines fitted to the two portions of the curve constructed from the TBZ binding data. The $n_{1}$ and $K_{1}$ values for the high affinity class of TBZ-binding sites were 0.14 and $40.7 \times 10^{4} \mathrm{l} / \mathrm{mol}$. The $n_{2}$ and $K_{2}$ values for the low affinity class of TBZ-binding sites were 0.86 and $3.4 \times 10^{4} 1 / \mathrm{mol}$. TBZ binding to spore surfaces appeared to be satisfactorily represented by these two classes of sites, the high affinity class comprising $14 \%$ of the total number of TBZ-binding sites on the spore surface while the low affinity class contributes $86 \%$ of the sites.

We explored the possibility that BIZ might share a class of binding sites in common with the substituted benzimidazoles. The u.v. spectrum of BIZ did not overlap the major absorption peaks of the substituted benzimidazoles; therefore their measurement was not hindered by the presence of BIZ. Spores were first mixed with BIZ for IO min; then a substituted benzimidazole was added. Only slight competition between BIZ and the substituted benzimidazoles for binding sites could be detected. For example, when spores were exposed to TBZ in the presence of an equimolar concentration of BIZ, no decrease in TBZ adsorption could be measured. When the BIZ concentration was raised 20 -fold relative to the $\mathrm{TBZ}$ concentration, there occurred only a $17.5 \%$ decrease in TBZ adsorption.

\section{DISCUSSION}

The removal of benzimidazole compounds from solution by Pithomyces chartarum spores is characteristic of a surface adsorption rather than an active uptake in the following respects: (i) the reaction is rapid, being virtually complete in I or $2 \mathrm{~min}$; (ii) dead spores appear as effective as live spores in the process; (iii) the system is temperature-independent over the normal biological range; (iv) the influence of ionic strength on the reaction (at least in the case of the unsubstituted benzimidazole) suggests ion-exchange properties for the system.

The $\mathrm{pH}$-dependency curves for adsorption had maxima at about $\mathrm{pH} 4.5$ and half-maxima at about $\mathrm{pH} 2.6$ and 6.8. These two latter values might represent the $\mathrm{p} K_{1}$ and $\mathrm{p} K_{2}$ values for the major adsorptive sites on the spore surface. The benzimidazoles themselves would act as cations at $\mathrm{pH}_{4.5}$ (BIZ and PBIZ have $\mathrm{p} K$ values of 5.4 and 5.2 respectively). This system differs from the uptake of BIZ by yeast cells (Slonimski, I956) where only the unionized BIZ was removed from solution.

BIZ adsorption differs from that of the three substituted benzimidazoles in several respects: (i) it is more affected by ionic strength; (ii) the theoretical maximal amount which can be adsorbed/mg dry wt of spores is only about one quarter to one half that of the substituted benzimidazoles; (iii) there is only one class of BIZ-adsorptive sites on the spore 
surface whereas there are at least two classes of sites for the substituted benzimidazoles. One class has a high affinity for the adsorbing molecules while the other has a relatively low affinity; in this regard, the binding is quite similar to that revealed by the Scatchard analysis of the binding of salicylate, bromsulphophthalein, and phenylbutazone to bovine serum albumin molecules (Rudman, Bixler \& Del Rio, 1971).

The surface adsorption of cations has been demonstrated in bacteria (McCalla, 1940), fungi (Rothstein \& Hayes, 1956), higher plants (Williams \& Coleman, I950) and erythrocytes (Abramson, Moyer \& Gorin, I942). In the present system the role which the adsorption plays in the eventual inhibition of Pithomyces chartarum spore germination by substituted benzimidazoles has yet to be determined. Perhaps adsorption serves to concentrate the benzimidazole compounds at the spore surface to act as a reservoir of inhibitor when spore dormancy is broken and permeability increases. Such a role would be of major importance in a stock-grazing area sprayed with TBZ to inhibit the germination of $P$. chartarum conidia. A dormant spore with its high surface affinity for TBZ could accumulate a lethal concentration even in the presence of other materials (such as plant tissue and soil) which might compete for free TBZ molecules.

We are grateful to Dr Margaret di Menna for the production of the spores used in this work, and to Dr E. P. White for critical evaluation of the manuscript. We particularly thank Dr John Watkinson for his assistance in the analysis of the adsorption isotherm data.

\section{REFERENCES}

Abramson, H. A., Moyer, L. W. \& Gorin, M. H. (1942). Electrophoresis of Proteins and the Chemistry of Cell Surfaces. New York: Reinhold Publishing Co.

Bertaud, W. S., Morice, I. M., Russell, D. W. \& Taylor, A. (I963). The spore surface in Pithomyces chartarum. Journal of General Microbiology 32, 385-395.

Bieber, S. \& Hitchings, G. H. (I955). The developing frog embryo and growth-inhibitors. Cancer Research Supplement 3, 80-82.

Brown, G. C. (1952). The influence of chemicals on the propagation of poliomyelitis virus in tissue culture. Journal of Immunology 69, 44I-450.

Campbell, A. G. (1969). Prevention of facial eczema in grazing stock. New Zealand Journal of Agriculture II9, 28-3I.

EdGington, L. V., KhEw, K. L. \& BARron, G. L. (197I). Fungitoxic spectrum of benzimidazole compounds. Phytopathology 61, 42-44.

Edsall, J. T. \& Wyman, J. (1958). Biophysical Chemistry, vol. I, p. 61 I. New York: Academic Press.

Hayward, D. O. \& Trapnell, B. M. W. (1964). Chemisorption, 2nd edn, pp. I59-168. London: Butterworths.

Horowitz, W. (1970). Official Methods of Analysis of the Association of Official Analytical Chemists, II th edn, pp. 750-75I. Washington, D.C.: Association of Official Analytical Chemists.

LANGmuir, I. (1916). The constitution and fundamental properties of solids and liquids. I. Solids. Journal of the American Chemical Society 38, $2221-2295$.

Lowry, R. J. \& Sussman, A. S. (1956). Physiology of the cell surface of Neurospora ascospores. II. Interference with dye adsorption by Polymyxin. Archives of Biochemistry and Biophysics 62, I 13-I 24.

MCCAlla, T. M. (1940). Cation adsorption by bacteria. Journal of Bacteriology 40, 23-32.

di Menna, M. E., Campbell, J. \& Mortimer, P. H. (I970). Sporidesmin production and sporulation in Pithomyces chartarum. Journal of General Microbiology 6r, 87-96.

Rothstein, A. \& HAYES, A. D. (1956). The relationship of the cell surface to metabolism. XIII. The cationbinding properties of the yeast cell surface. Archives of Biochemistry and Biophysics 63, 87-99.

Rudman, D., Bixler, T. J. \& Del Rio, A. E. (1971). Effect of free fatty acids on binding of drugs by bovine serum albumin, by human serum albumin, and by rabbit serum. Journal of Pharmacology and Experimental Therapeutics $\mathbf{1 7 6}, 261-272$. 
SCATCHARD, G. (1949). The attraction of proteins for small molecules and ions. Annals of the New York Academy of Science 51, 660-672.

Sinclair, D. P. \& Howe, M. W. (I968). Effect of thiabendazole on Pithomyces chartarum (Berk. \& Curt.) M. B. Ellis. New Zealand Journal of Agricultural Research Ir, 59-62.

SLonimski, P. P. (1956). Penetration du benzimidazole dans la cellule de levure. Experimental Cell Research Io, $160-167$.

Sussman, A. S., von Boventer-Heidenhain, B. \& Lowry, R. J. (1957). Physiology of the cell surface of Neurospora ascospores. Plant Physiology 32, 586-590.

Williams, D. E. \& Coleman, N. T. (1950). Cation exchange properties of plant root surfaces. Plant and Soil 2, 243-256.

Woolley, D. W. (1944). Some biological effects produced by benzimidazole and their reversal by purines. Journal of Biological Chemistry 152, 225-232. 